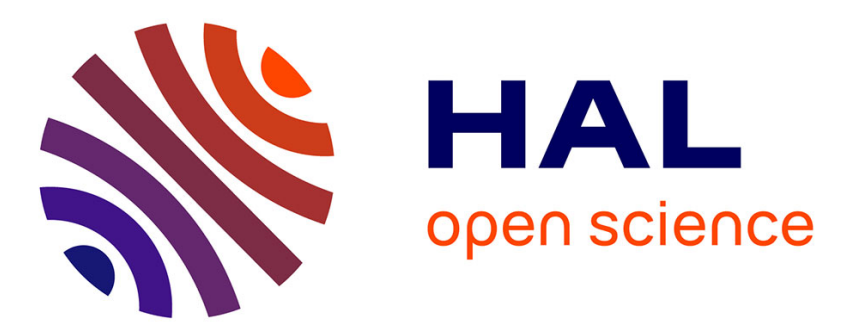

\title{
Soustraction des bruits de fond basée sur l'estimation du spectre à court terme. Réalisation en temps réel
}

\author{
H. Zeng
}

\section{To cite this version:}

H. Zeng. Soustraction des bruits de fond basée sur l'estimation du spectre à court terme. Réalisation en temps réel. Journal de Physique IV Proceedings, 1994, 04 (C5), pp.C5-1331-C5-1334. 10.1051/jp4:19945296 . jpa-00253009

\section{HAL Id: jpa-00253009 https://hal.science/jpa-00253009}

Submitted on 1 Jan 1994

HAL is a multi-disciplinary open access archive for the deposit and dissemination of scientific research documents, whether they are published or not. The documents may come from teaching and research institutions in France or abroad, or from public or private research centers.
L'archive ouverte pluridisciplinaire HAL, est destinée au dépôt et à la diffusion de documents scientifiques de niveau recherche, publiés ou non, émanant des établissements d'enseignement et de recherche français ou étrangers, des laboratoires publics ou privés. 


\title{
Soustraction des bruits de fond basée sur l'estimation du spectre à court terme. Réalisation en temps réel
}

\author{
H. ZENG
}

Ministère de la Culture, Université Paris VI, URA 879 du CNRS, Laboratoire de Mécanique Physique, Le Val Joyeux CD11, 78450 Villepreux, France

\begin{abstract}
In order to restore degraded audio recordings in real time for French National Library, we developed a parallel structure system with PC-based multiprocessor Motorola DSP96002. The study is for some methods of speech enhancement using short-time spectral amplitude estimation. The rapidity and flexibility of our system programme for WINDOWS give us the possibility to realise many applications in sound analysis and synthesis with great performance.
\end{abstract}

Résumé : Afin de permettre à la Bibliothèque Nationale la restauration en temps réel d'enregistrements sonores dégradés, une structure parallèle basée sur multiprocesseurs Motorola DSP96002 est développée. L'étude porte sur les méthodes de soustraction des bruits par l'estimation du spectre à court terme. La rapidité et la souplesse d'une structure gérée par notre programme pour WINDOWS offrent la possibilité de réaliser sur $\mathrm{PC}$ un système adaptatif très performant pour ce type de traitement.

\section{Introduction.}

Parmi les méthodes de soustraction de bruits par l'estimation du spectre à court terme, un bon nombre sont arrivés à des résultats intéressants. Mais, par suite de la complexité de l'algorithme et des difficultés propres aux matériels, ces études d'estimateurs sont plutôt restées au stade de la théorie.

Dans notre projet de système de restauration d'enregistrements sonores dégradés pour la Bibliothèque Nationale( qui s'inscrit dans le cadre d'une collaboration avec le Laboratoire d'Acoustique du Mans), un multiprocesseur constitué de plusieurs cartes DSP96002 a été réalisé. Grâce à șa vitesse de calcul et à sa souplesse de communication, cette structure parallèle nous permet de réaliser, en temps réel, les traitements de ce type dans un PC. La technique de programmation pour WINDOWS nous a aidé à réaliser nos travaux de manière très souple, avec une bonne qualité de l'interface homme-machine.

\section{Réalisation}

Nous avons choisi une structure parallèle, constituée de plusieurs cartes processeurs DSP96002 reliées par un bus Motorola dans un PC[1]. Elle comporte deux voies entrée et sortie et deux convertisseurs de précision 16 bits. Sa puissance totale, pour un maximum de 
quatre cartes est de 200 MFLOPS. Le système basé sur ce multiprocesseur est schématisé sur la Fig.1.

La première carte DSP-1 du multiprocesseur est consacrée à la réalisation de la boucle de base du traitement, y compris les fonctions suivantes: 1). gestion d'entrée et sortie; 2 ). tampon d'entré et sortie; 3). pré-traitement; 4). fenêtre de Hanning; 5). FFT $\times 2 ; 6$ ). séparation de 2 FFT; 7). multiplication des coefficients; 8). FFT inverse; 9). reconstitution (ou synthèse) du signal.

L'ensemble des fonctions gérées par le système est contrôlé par l'intermédiaire de WINDOWS 3.1 sur le PC. Les fonctions principales sont $: 1$ ). affichage en temps réel du signal pour surveiller les procédures de traitement; 2 ). analyse et impression graphique du signal pour comparer, évaluer et enregistrer les résultats; 3 ). analyse sonographique et réécoute de la reconstitution du son en suivant le déplacement du curseur d'indication; 4). simulation ou constitution du filtrage en temps réel, y compris les fonctions d'inventaire et sauvegarde des échantillons de défauts rencontrés en traitant les enregistrements sonores; 5). enregistrement des signaux sur le disque dur; 6). panneau de configuration, qui commande et gère l'ensemble du système.

Le traitement des algorithmes, des estimateurs de spectre à court terme, est réalisé parallèlement par les processeurs DSP2, 3 et 4. Le nombre de cartes parallèles est choisi en fonction de la complexité de l'estimateur et de la vitesse demandée.

\subsection{Augmentation de la Rapidité}

Traitement en parallèle: La gestion d'entrée et sortie, les communications entre les DSP et le PC assurées par la "dual-port memory", les communications entre les DSP assurées par les mémoires globales du multiprocesseur et les traitements de l'estimateur sont effectués sous les programmes d'interruption de chaque processeur.

Amélioration des algorithmes : on double la rapidité de la FFT en la calculant une fois pour deux sections de données réelles[2]. L'amélioration de la rapidité des algorithmes en fonction des caractéristiques du DSP est recherchée dans toutes les procédures de traitement.

\subsection{Améliorations de la Souplesse}

Fenêtre et synthèse: La fenêtre de Hanning est réglable librement entre la longueur et la demi-longueur de la FFT. Les sections de données sont acquises l'une après l'autre par déplacement d'une demi-longueur de la fenêtre dans le tampon d'entrée-sortie. Ensuite le signal est restauré par recouvrement des sections traitées, dans les mêmes conditions que l'acquisition.

Gain : Cette structure sépare les applications et le système de base. La seule chose à faire pour les développements ultérieurs est le calcul des coefficients du gain, ce qui permet de développer des applications plus facilement et sans avoir à connaître le système de base. Des applications sont développées. La simulation du filtrage assure le chargement continu des coefficients dans le DSP quand on déplace la souris pour dessiner un profil de filtre sur l'écran. Le chargement d'un groupe de coefficients sélectionné dans une liste nous donne la possibilité de supprimer le bruit de fond stationnaire connu rencontré dans des enregistrements sonores.

Combinaison des fonctions : Toutes les fonctions dans la boucle de base du traitement sont sous le contrôle du panneau de configuration. Des combinaisons de fonctions nécessaires pour une tâche peuvent être réalisées en activant et désactivant la fonction correspondante dans la boîte de dialogue. Par exemple, pour téaliser un sonogramme du signal venant directement du convertisseur, sélectionner les fonctions de boucle de base $1,2,4,5,6$ et 7; pour reconstituer le signal d'un sonographe, sélectionner les fonctions $10,11,2$ et 1 , etc. 


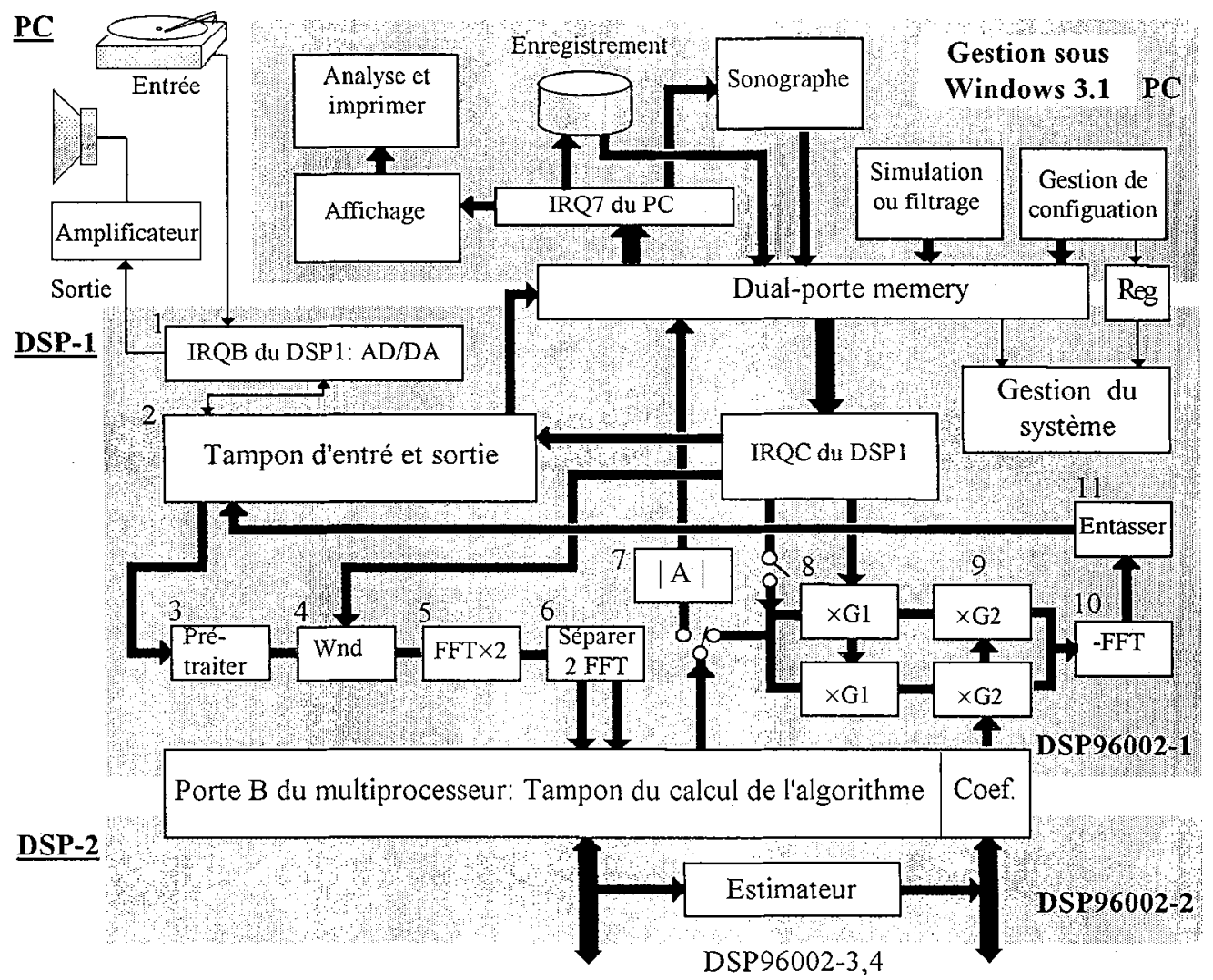

Figure 1 Multiprocesseur Système de Restauration des Enregistrements Sonores

\section{Performances et Résultats}

Avant le développement de l'estimateur plus complexe proposé par J.C.VALIERE [3], un algorithme simple mais efficace, proposé par STEVEN F. BOLL[4], a été choisi pour essayer le système de base. Dans cette démonstration, nous utilisons deux cartes DSP96002 (au lieu de quatre) pour éprouver la structure parallèle du multiprocesseur. Ce test s'est avéré suffisant pour toutes les applications avec quatre cartes DSP, car les DSP-2,3,4 sont synchronisées par le DSP-1.

Dans nos présentations, $\hat{S}\left(e^{j w}\right)$ est l'élément du signal à estimer, $X\left(e^{j w}\right)$ est élément du spectre à traiter, $N\left(e^{j \omega}\right)$ est le bruit, l'algorithme est

$$
\hat{S}\left(e^{j \sigma}\right)=H\left(e^{j \omega}\right) X\left(e^{j \omega}\right) \text { avec } H\left(e^{j \omega}\right)=1-\frac{\mu\left(e^{j \omega}\right)}{\left|X\left(e^{j \omega}\right)\right|}, \mu\left(e^{j \omega}\right)=E\left\{\left|N\left(e^{j \omega}\right)\right|\right\} .
$$

Ici, $\mu\left(e^{j \omega}\right)$ est la moyenne d'éléments $N\left(e^{j \omega}\right)$ échantillonnés dans un intervalle de silence entre les séquences de parole ou de musique.

Dans notre essai, le signal original bruité par le secteur, et mélangé avec le signal carré $(300 \mathrm{~Hz})$ d'un générateur, est choisi comme source(Fig.2a et Fig.3 en haut). Après traitement, le bruit est supprimé parfaitement (Fig.2b et Fig. 3 en bas). 
De plus, nous avons ajouté un filtrage réglable en temps réel, utilisable comme une console de studio et géré sous WINDOWS.

Performances : La longueur de la fenêtre de Hanning est réglable continûment de 32 points jusqu'à 1024 points. La fréquence d'échantillonnage du système est de $32 \mathrm{kHz}$ pour une longueur de FFT de 1024 points avec une seule carte DSP96002(elle peut arriver jusqu'à $400 \mathrm{kHz}$ sans traitement) La fréquence d'échantillonnage, pour enregistrer deux voies de signaux sur disque dur sous WINDOWS, est de $40 \mathrm{kHz}(80 \mathrm{kHz}$ sous DOS).

\section{Conclusion}

Le système développé dans ce travail est une structure parallèle consacrée au traitement en temps réel; sa rapidité et sa souplesse permettront ultérieurement de développer des applications d'analyse et de synthèse du son. Le nombre de cartes peut être choisi en fonction de la complexité de l'algorithme. Ce système permet l'échange de données entre les processeurs sans interrompre le traitement. La réalisation d'un réglage adaptatif des paramètres du système est possible pour des applications plus performantes.

\section{Références}

[1] Loughborough Sound Images Ltd, "DSP96002 Technical Reference Manual", England

[2] E. ORAN BRIGHAM, "The Fast Fourier Transform" Prentice-Hall, Inc. Englewood Cliffs, New Jersey 1974

[3] J.C.VALIERE, Thèse de l'Université du Maine, 1991

[4] STEVEN F. BOLL, "Suppression of Acoustic Noise in Speech Using Spectral Subtraction" IEEE Trans. Acoust., Speech, Signal Processing, vol. ASSP-27, pp. 113-120, APRIL 1979

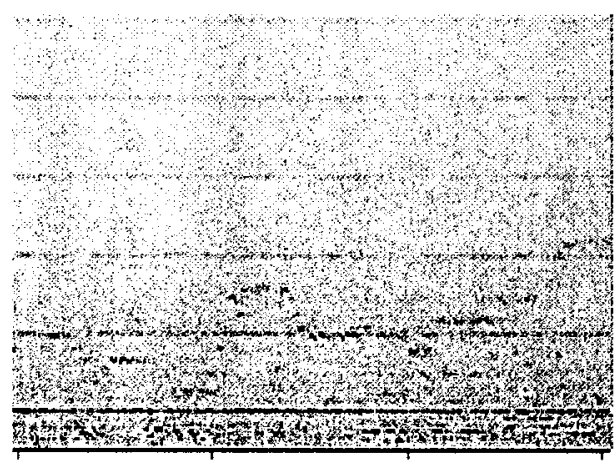

Fig. 2a, Signal bruité par le signal carré stationnaire avec bruit de fond du lecteur de cassette.

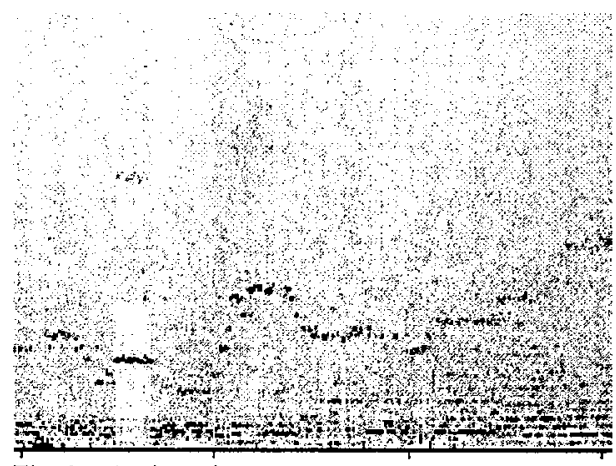

Fig. 2a, Après traitement.

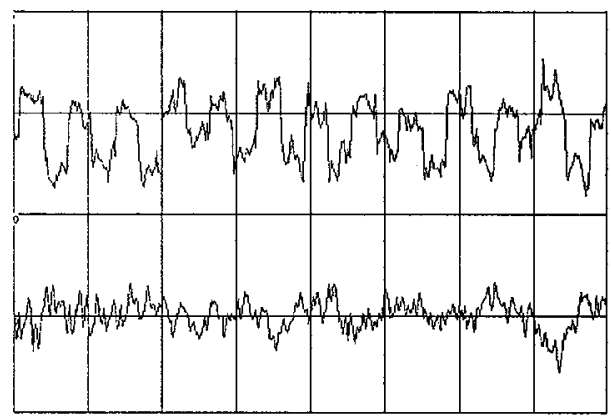

Fig.3 En haut - signal musique bruité par signal carré stationnaire et bruit de fond.

En bas - sortie du traitement, après suppression des bruits. 